\title{
EFFECT OF THE MYELIN SHEATH AQUEOUS LAYERS ON THE ACTION POTENTIALS IN SIMULATED HEREDITARY AND CHRONIC DEMYELINATING NEUROPATHIES
}

\author{
Krustev S. ${ }^{1}$, D. Stephanova ${ }^{2}$ \\ ${ }^{1}$ Department of Medical Physics and Biophysics, Faculty of Pharmacy, \\ Medical University of Varna, ${ }^{2}$ Institute of Biophysics and Biomedical Engineering, \\ Bulgarian Academy of Sciences, Sofia
}

\begin{abstract}
The action potentials reflect the conduction properties in nerve excitable structures. In order to expand our studies of the conduction properties, the effect of the myelin sheath aqueous layers on propagated action potentials is compared in previously simulated by us demyelinating neuropathies such as Charcot-Marie-Tooth disease type 1A (CMT1A), chronic inflammatory demyelinating polyneuropathy (CIDP) and CIDP subtypes. Using our multi-layered model of human motor nerve fibre, the calculated action potentials are presented in a simulated normal case and in three cases of progressively greater demyelinations (i. e. internodal systematic demyelinations (ISDs) as specific indicators of CMT1A; paranodal systematic demyelinations (PSDs) as specific indicators of CIDP; and paranodal internodal systematic demyelinations (PISDs) - as specific indicators of CIDP subtypes for $s=1,2,3$ ) without/with aqueous layers within the myelin sheath. Two cases of each demyelinated subtype are mild and one is severe. The loss of the myelin lamellae and their corresponding aqueous layers in the ISD1 and ISD2 cases additionally slows the conduction velocity. The uniform reduction of the paranodal seal resistance in the PSD1 and PSD2 cases with aqueous layers additionally increases the conduction velocity of the action potentials in comparison with the cases when the aqueous layers are not taken into account. However, the effect of the aqueous layers on the conduction properties is neutralized when the demyelinations are heterogeneous such as in the PISDs. The present study shows that the conduction velocity of the action potentials in the simulated CMT1A and CIDP depends on the aqueous layers.
\end{abstract}

Key words: myelin sheath aqueous layers, action potential, demyelinating neuropathies, computational neuroscience

\section{INTRODUCTION}

Studies of native myelin by X-ray and neutron diffraction have demonstrated that the myelin lamellae are not tightly compacted, but separated by cytoplasmic and extracellular spaces of about 4-5 nm, respectively, so that up to half the volume fraction of myelin is taken up by water $(5,6)$. The present study compares the effect of the aqueous layers on the previously calculated action potentials, reflecting the propagating processes in simulated by us demyelinating neuropathies such as Charcot-Marie-Tooth disease type 1A (CMT1A), chronic inflammatory demyelinating polyneuropathy (CIDP) and its subtypes $(9,14,15)$. CMT1A is the most common form of hereditary neuropathy. Its hallmark is uniform demyelination, which results in slowing the conduction in motor and sensory nerves $(1,11)$.

Address for correspondence:

D. Stephanova, Institute of Biophysics and Biomedical Engineering,

Bulgarian Academy of Sciences,

Acad. G. Bontchev Str., Bl. 21, Sofia 1113, Bulgaria

e-mail: dsteph@shiva.bio.bas.bg
CIDP as a peripheral nerve disorder is one of several chronic demyelinating neuropathies that are believed to have autoimmune etiology (7). There are subtypes of chronic demyelinating neuropathies $(8,10)$ classified under the term of chronic inflammatory demyelinating polyneuropathy.

\section{MATERIAL AND METHODS}

The computations use our model (13), in which 150 interconnected parallel lamellae are simulated by alternating 150 lipid and 150 aqueous layers within the myelin sheath. All calculations are carried out for fibres with: an axon diameter of $12,5 \mu \mathrm{m}$; an external fibre diameter of $17,3 \mu \mathrm{m}$; nodal diameter of $5 \mu \mathrm{m}$; nodal area of $24 \mu \mathrm{m}^{2}$; myelin thickness of $2,4 \mu \mathrm{m}$; periodicity of myelin lamellae of 16 $\mathrm{nm}$, and periaxonal space thickness of $20 \mathrm{~nm}$. The temperature is $37^{\circ} \mathrm{C}$.

Schematic diagrams of normal case and of the systematic demyelinations each of them internodal, paranodal and paranodal internodal are presented in Fig. 1. The reduction 
of the myelin lamellae (defining internodal demyelination), or of the paranodal seal resistance (defining paranodal demyelination), or simultaneously both of them (defining paranodal internodal demyelination) is uniform along the fibre length for the systematically demyelinated subtypes. These permutations are termed internodal systematic demyelination (ISD), paranodal systematic demyelination (PSD), and paranodal internodal systematic demyelination (PISD), respectively. Three progressively greater degrees (two mild - 70\%, 80\% and one severe) of each of the systematic demyelinations (i. e. ISDs, PSDs and PISDs for $\mathrm{s}=1,2,3$ ) are investigated without/with taking into account the aqueous layers within the myelin sheath.

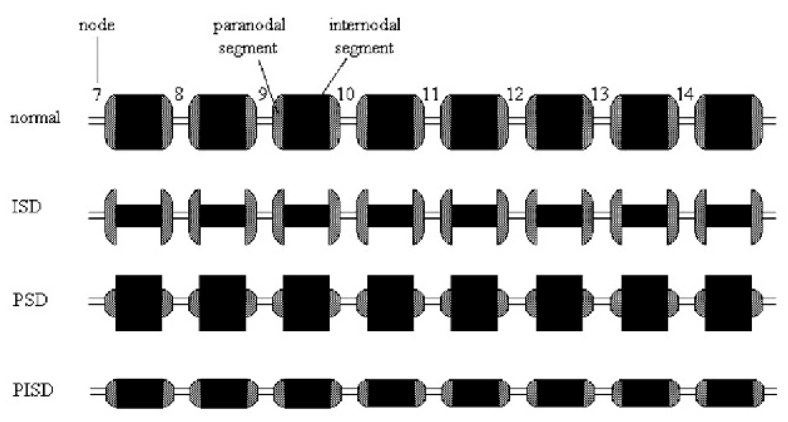

Fig. 1. Diagram of human motor nerve fibres from the $7^{\text {th }}$ to the $14^{\text {th }}$ nodes in the normal, ISD, PSD and PISD cases. Three progressively greater degrees (two mild $70 \%, 80 \%$ and one severe) of each systematic demyelination (i. e., ISD1, ISD2, ISD3; PSD1, PSD2, PSD3; PISD1, PISD2 and PISD3) are simulated without/with myelin sheath aqueous layers

The $70 \%$ (ISD1, PSD1 and PISD1) and $80 \%$ (ISD2, PSD2 and PISD2) values of the myelin lamellae that are uniformly reduced along the fibre length are not sufficient to develop a conduction block of the intracellular action potential. Such demyelinations are regarded as mild. The reduction values of $93 \%$ (ISD3) $/ 93 \%$ (ISD3); $90 \%$ (PSD3) $/ 89 \%$ (PSD4), and $82 \%$ (PISD3)/82\% (PISD3) are the first degrees of the subtypes for achieving the conduction block in the given demyelinated cases with/without taking into consideration the aqueous layers within the myelin sheath, respectively. Such demyelinations are regarded as severe. The comparison between the action potentials without and with taking into account the aqueous layers within the myelinated sheath reveals the effect of the aqueous layers on the investigated axonal conduction properties. The $70 \%$ and $80 \%$ reduction values of the myelin lamellae are not arbitrarily taken. It was previously found by as that the direction of the longitudinal current flow is the same as in the axoplasm from the $2^{\text {nd }}$ to the $25^{\text {th }}$ aqueous layer, whereas to the $50^{\text {th }}$ lamella this direction is reversed and above the $75^{\text {th }}$ lamella the longitudinal myelin currents are negligible (13).

The myelin reduction value of a given subtype for achieving the conduction block depends on the type of demyelinations. It ranges from $93 \%$ to $82 \%$ for the ISD3 and PISD3, respectively. These reduction values are same for the ISD3 and PISD3 subtypes without and with myelin aqueous layers. However, these values are different for the paranodal systematic demyelinations. Note the $90 \%$ myelin reduction value for the PSD3 case with aqueous layers and the $89 \%$ myelin reduction value for the PSD4 case without aqueous layers. The results presented here are consistent with the interpretation that: (i) genetic factors causing changes in the internodal segments of the myelin sheath, without or with aqueous layers; as well as (ii) immunological factors causing changes in the paranodal segments of the myelin sheath, without or with aqueous layers; and (iii) simultaneously both of these factors causing changes in the myelin sheath, without or with aqueous layers, could be responsible for the axonal conduction abnormalities obtained in the simulated hereditary (CMT1A) and chronic (CIDP, CIDP subtypes) demyelinating neuropathies, respectively. The action potential stimulation is simulated by adding a short $(0,1 \mathrm{~ms})$ rectangular depolarizing current pulse to the centre of the first node. This case of intraaxonal point application of current at the node closely approximates the effects of extraaxonal point application of current at the node and realizes a point fibre polarization.

\section{RESULTS}

Comparison of the action potentials for the normal (first row), ISDs (a), PSDs (b) and PISDs (c) cases without (dotted lines) and with (continued lines) aqueous layers within the myelin sheath is shown for human motor nerve fibres (Fig. 2). The potentials are presented at the $10^{\text {th }}$ node only. The potential maxima at the $10^{\text {th }}$ nodes are $38 / 38,18 / 17$, $11 / 9 \mathrm{mV}$ in the normal, ISD1 and ISD2 cases without/with aqueous layers, respectively (Fig. 2a). A decrease of the conduction velocities is obtained for these abnormal cases, except for the normal case where the aqueous layers increase the conduction velocity by $8,6 \%$. The normal conduction velocity is of $58 \mathrm{~m} / \mathrm{s}$ without aqueous layers. The conduction velocities calculated from the times of the potential maxima at the nodes are of 31 and $23 \mathrm{~m} / \mathrm{s}$ for the ISD1 and ISD2 cases without aqueous layers, respectively. The velocity remains almost unchanged $(30,6 \mathrm{~m} / \mathrm{s})$ in the ISD 1 case and decreases by $3,4 \%$ in the ISD 2 case when the aqueous layers are taken into account. The progressively greater increase in uniform loss of the myelin lamellae without/with their aqueous layers blocks the invasion of the potentials in the ISD3 case. Thus, with the increase of the demyelination from the ISD1 to the ISD3, the conduction failure occurs rapidly. The reduction value of the myelin lamellae for achieving conduction block is the same for both cases without and with aqueous layers within the myelin sheath.

The maximal amplitudes are of $21 / 23$ and $12 / 13 \mathrm{mV}$ in the PSD1 and PSD2 cases without/with aqueous layers, respectively (Fig. $2 b$ ). The conduction velocities calculated from the times of the potential maxima at the nodes are 41 and $35 \mathrm{~m} / \mathrm{s}$ for the PSD1 and PSD2 cases without aqueous 

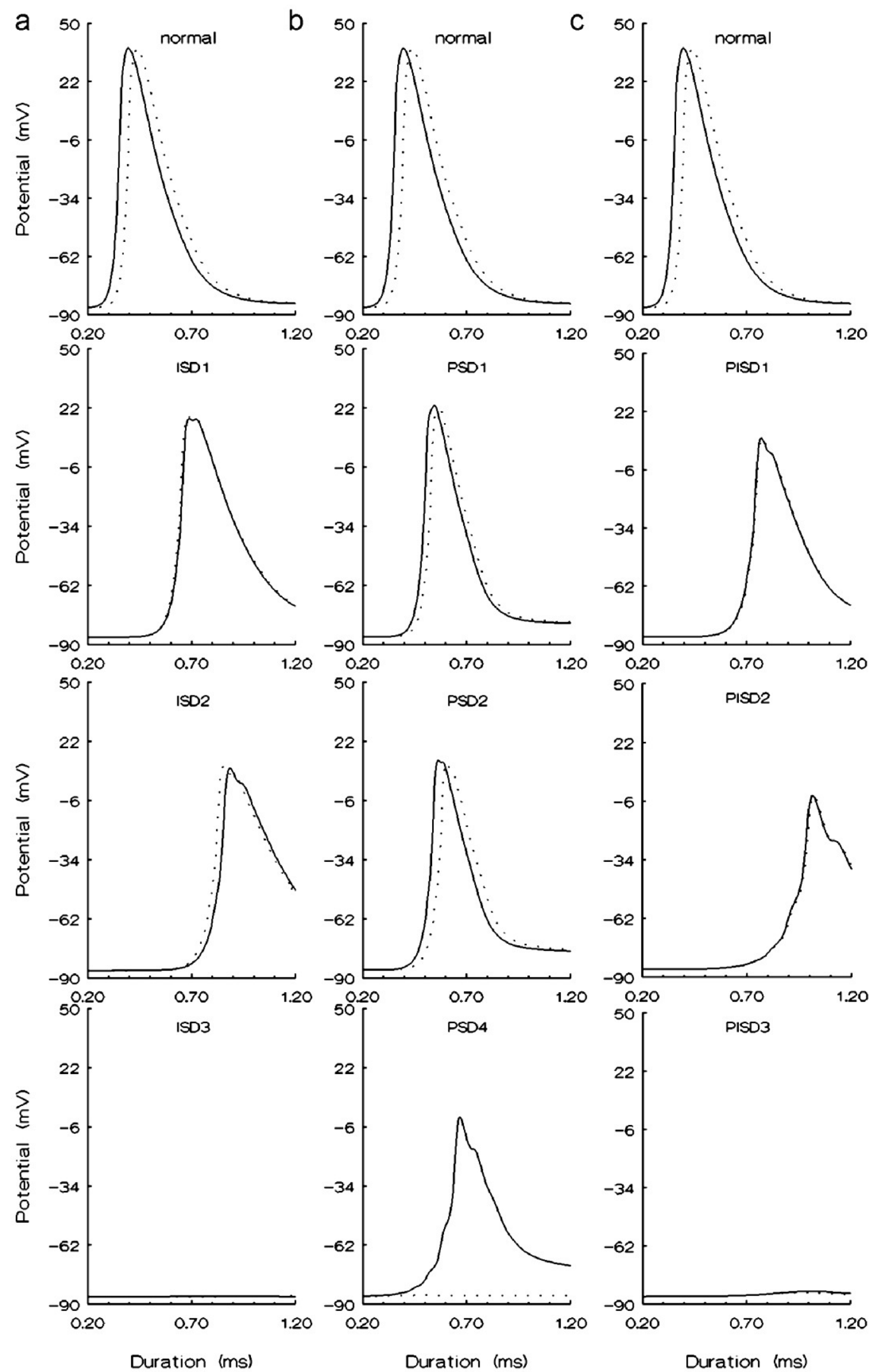

Fig. 2. Comparison between the action potentials of human motor nerve fibres in the normal, ISDs (a), PSDs (b) and PISDs (c) cases without aqueous layers presented by dotted lines and those with aqueous layers presented by continued lines. The potentials are given at $10^{\text {th }}$ node only. The aqueous layers in the $89 \%$ demyelinated case (PSD4) restore the action potential propagation, which is initially blocked when the aqueous layers are not taken into account 
layers, respectively. For the PSD1 and PSD2 cases, the addition of the aqueous layers increases the conduction velocities by 6,9 and $8,6 \%$, respectively. The progressively greater increase in uniform reduction of the paranodal seal resistance blocks the invasion of the potentials in the severe PSD3 case, when the aqueous layers are taken into account. Significantly, the aqueous layers in the $89 \%$ demyelinated case (PSD4) restore the action potential propagation (Fig. $2 \mathrm{~b}$, continued line - in the bottom), which was initially blocked when the aqueous layers were not taken into account (Fig. 2b, dotted line in the bottom). These data show that the aqueous layers markedly promote action potential propagation in normal cases and in cases of up to $89 \%$ paranodal demyelination. The maximal amplitude of the restored potential is of $-1,5 \mathrm{mV}$ and its conduction velocity is of $19 \mathrm{~m} / \mathrm{s}$.

An overlap of the action potentials at the nodes is obtained for all PISDs cases, without and with aqueous layers within the myelin sheath (Fig. 2c). The maximal amplitudes of the potentials are of 9 and $-3 \mathrm{mV}$ for the PISD1 and PISD2 cases, respectively. The conduction velocities are 25 and 13 $\mathrm{m} / \mathrm{s}$ for the PISD1 and PISD2 cases, respectively.

\section{DISCUSSION}

The results presented here show that the aqueous layers within the myelin sheath modulate the action potentials of human motor nerve fibres when the systematic demyelinations are internodal and paranodal. The loss of the myelin lamellae and their corresponding aqueous layers in the ISD1 and ISD2 cases additionally slows the conduction velocity of action potentials in comparison with the cases when the aqueous layers are not taken into account. The conduction velocities are in the range of 31/30,6 $23 / 22,2 \mathrm{~m} / \mathrm{s}$ for the cases without/with myelin aqueous layers, respectively. The mean median nerve conduction velocities measured in patients with CMT1A are of $21 \pm 5,7$ $\mathrm{m} / \mathrm{s}(4)$ and of $16,5 \mathrm{~m} / \mathrm{s}$ in the range of $5-35 \mathrm{~m} / \mathrm{s}(3,12)$. The relatively weak effect of the aqueous layers on the propagating processes in the investigated ISD1 and ISD2 cases can account for the same reduction values $(93 \% / 93 \%)$ of the myelin lamellae without/with aqueous layers, respectively, obtained for the conduction block.

The uniform reduction of the paranodal seal resistance in the PSD1 and PSD2 cases, with taking into account the aqueous layers within the myelin sheath, additionally increases the conduction velocity of the action potentials in comparison with the cases when the aqueous layers are not taken into consideration. The calculated conduction velocities are in the range of $41 / 44-35 / 38 \mathrm{~m} / \mathrm{s}$ for the cases without/with aqueous layers, respectively. The conduction velocities in patients with CIDP measured in median motor axons in the elbow-wrist segment are in the range of 54-20 $\mathrm{m} / \mathrm{s}(2,16)$. For the one severely demyelinated case, for which propagation without aqueous layers was blocked, the aqueous layers restore the action potential propagation.
The conduction velocity of action potential in the case just before the blockage (PSD4 of $89 \%$ ) is of $19 \mathrm{~m} / \mathrm{s}$.

The results presented here also show that the aqueous layers within the myelin sheath do not modulate the action potentials of human motor fibres when the demyelinations are simultaneously paranodal internodal. Consequently, the effect of the aqueous layers on the conduction properties is neutralized when the demyelinations are heterogeneous such as in the PISDs. The changes in the potentials obtained in the PISDs simulations are in good accordance with the data from patients with CIDP subtypes (16).

\section{REFERENCES}

1. Birouk, N., R. Gouider, E. Le Guern, M. Gugenheim, S. Tardieu, T. et al. Charcot-Marie-Tooth disease type $1 \mathrm{~A}$ with $17 \mathrm{p} 11.2$ duplication. Clinical and electrophysiological phenotype study and factors influencing disease severity in 119 cases.Brain, 120, 1997, No 5, 813-823.

2. Cappelen-Smith, C., S. Kuwabara, C. S.-Y Lin, I. Mogyoros, D. Burke. Membrane properties in chronic inflammatory demyelinating polyneuropathy.- Brain, 124, 2001, No 12, 2439-2447.

3. Carvalho, A. A., A. Vital, X. Ferrer, P. Latour, A. Lagueny, C. Brechenmacher, et a1. Charcot-Marie-Tooth disease type 1A: clinicopathological correlations in 24 patients.- $J$. Peripher. Nerv. Syst., 10, 2005, No 1, 85-92.

4. Hattori, N., M. Yamamoto, T. Yoshihara, H. Koike, M. Nakagawa, H. Yoshikawa, et al.; Study Group for Hereditary Neuropathy in Japan. Demyelinating and axonal features of Charcot-Marie-Tooth disease with mutations of myelin-related proteins (PMP22, MPZ and Cx32): a clinicopathologi-cal study of 205 Japanese patients.Brain, 126, 2003, No 1, 134-151.

5. Kirschner, D. A., D. L. Caspar. Comparative diffraction studies on myelin membrane.- Ann. N. Y. Acad. Sci., 195, 1972, 309-317.

6. Kirschner, D. A., A. L. Ganser, D. L. Caspar. Diffraction studies of molecular organization and membrane interactions in myelin.- In: Myelin. P. Morrel ed. $2^{\text {nd }}$ ed. New York, Plenum Press, 1984, 51-59.

7. Köller, H., B. C. Kieseier, S. Jander, H. P. Hartung. Chronic inflammatory demyelinating polyneuropathy.- New Engl. J. Med., 352, 2005, No 13, 1343-1356.

8. Krarup, C., J. D. Stewart, A. J. Sumner, A. Pestronk, S. A. Lipton. A syndrome of asymmetric limb weakness with motor conduction block.Neurology, 40, 1990, No 1, 118-127.

9. Krustev, S., M. Daskalova, D. Stephanova. Myelin sheath aqueous layers do not modulate membrane fibre properties of simulated cases of paranodal internodal systematic demyelinations.- Compt. Rend. Acad Bulg. Sci, 63, 2010, No 12, 1845-1852.

10. Lewis, R. A., A. J. Sumner, M. J. Brown, A. K. Asbury. Multifocal demyelinating neuropa- 
thy with persistent conduction block.- Neurology, 32, 1982, No 9, 958-964.

11. Lupski, J. R., R. Montes de Oca-Luna, S. Slaugenhaupt, L. Pentao, V. Guzzetta, B. J. Trask, et al. DNA duplication associated with Charcot-Marie-Tooth disease type 1A.- Cell, 66, 1991, No 2, 219-232.

12. Marques, W. Jr, M. R. Freitas, O. J. Nascimento, A. B. Olivera, L. Calia, A. Melo, et al. 17p duplicated Charcot-Marie-Tooth 1A: characteristics of a new population.- J. Neurol., 252, 2005, No 8, 972-979.

13. Stephanova, D. I. Myelin as longitudinal conductor: a multi-layered model of the myelinated human motor nerve fibre.- Biol. Cybern., 84, 2001, No 4, 301-308.
14. Stephanova, D. I., S. M. Krustev, M. Daskalova. The aqueous layers within the myelin sheath modulate the membrane properties of simulated hereditary demyelinating neuropathies.- $J$. Integr. Neurosci., 10, 2011, No 1, 89-103.

15. Stephanova, D. I., S. M. Krustev, N. Negrev, M. Daskalova. The myelin sheath aqueous layers improve the membrane properties of simulated chronic demyelinating neuropathies.- J. Integr. Neurosci., 10, 2011, No 1, 105-120.

16. Sung, J. Y., S. Kuwabara, R. Kaji, K. Ogawara, M. Mori, K. Kanai, et al. Threshold electrotonus in chronic inflammatory demyelinating polyneuropathy: correlation with clinical profiles.- Muscle Nerve, 29, 2004, No 1, 28-37. 